\title{
The Performance of the Transition Radiation Detector of the AMS-02 experiment
}

\author{
Simonetta Gentile, \\ Università di Roma,"La Sapienza”, Sezione di Romal dell'INFN, Roma, Italy \\ on behalf of AMS02-TRD collaboration \\ I.Phys.Inst. RWTH Aachen,Germany, Sezione di Romal dell'INFN, Roma, Italy, IEKP Univ. of Karlsruhe, \\ Germany, Laboratory for Nuclear Science, MIT,Cambridge, MA, USA, \\ Kyungpook National University, Taegu, Korea
}

\begin{abstract}
The Alpha Magnetic Spectrometer (AMS-02) is an experiment which will be mounted on the International Space Station (ISS) in 2006 to measure primary cosmic ray spectra in space and to perform an indirect search of dark matter component of universe. A key element is a Transition Radiation Detector (TRD) to distinguish an $\mathrm{e}^{+}$or $\mathrm{p}^{-}$signal reducing the $\mathrm{p}^{+}$or $\mathrm{e}^{-}$background by a rejection factor $10^{-3}-10^{-2}$ in an energy range from 10-300 $\mathrm{GeV}$. This will be used in conjunction with an electromagnetic calorimeter to provide overall $\mathrm{p}^{+}$rejection of $10^{-6}$ at $90 \% \mathrm{e}^{+}$efficiency.

The detector consists of 20 layers of $6 \mathrm{~mm}$ diameter straw tubes alternating with $20 \mathrm{~mm}$ layers of polyethylene/polypropylene fleece radiator. The tubes are filled with a 80\%:20\% mixture of $\mathrm{Xe}: \mathrm{CO}_{2}$ at 1.0 bar absolute from a recirculating gas system designed to operate $>3$ years in space.

A TRD protype has been calibrated and its performance measured in test beams with $\mathrm{p}^{+}, \mathrm{e}^{-}, \mu^{-} \pi^{-}$in the momentum range from 3 to $250 \mathrm{GeV} / \mathrm{c}$ and compared with Monte-Carlo predictions. It achieved a rejection factors from 2000-140 for protons in an momentum range of $15-250 \mathrm{GeV} / \mathrm{c}$. The design and construction of the detector is presented and results from test beam runs are discussed.
\end{abstract}

Index Terms-AMS, TRD, transition radiation detector, proton rejection.

\section{INTRODUCTION}

$\mathbf{T}$ HE Alpha Magnetic Spectrometer is an experiment which will be mounted on the International Space Station (ISS) to measure primary cosmic ray spectra in space [1]. A main physics goal of AMS-02 is the search for dark matter. One way of doing this is to search for an enhancement in the positron spectrum as a function of energy. Since the ratio of fluxes of $\mathrm{p}$ to $\mathrm{e}^{+}$in orbit is on the order of $10^{4}$, AMS- 02 must be able to avoid confusing protons with positrons to a level better than $10^{-6}$. Its uppermost element is a Transition Radiation Detector (TRD) (see Fig. 1). Transition radiation (TR) consists of soft $\mathrm{X}$-rays which are emitted when charged particles traverse the boundary between two media with different dielectric constants. In the momentum range of $10-300 \mathrm{GeV} / \mathrm{c}$, light particles such as electrons and positrons have much higher probability of emitting TR photons than heavy particles such as protons and antiprotons. At a single boundary, the probability of emission is still very small, on the order of $10^{-2}$, but this is enhanced by using a fleece as a radiator. This, in turn is divided into

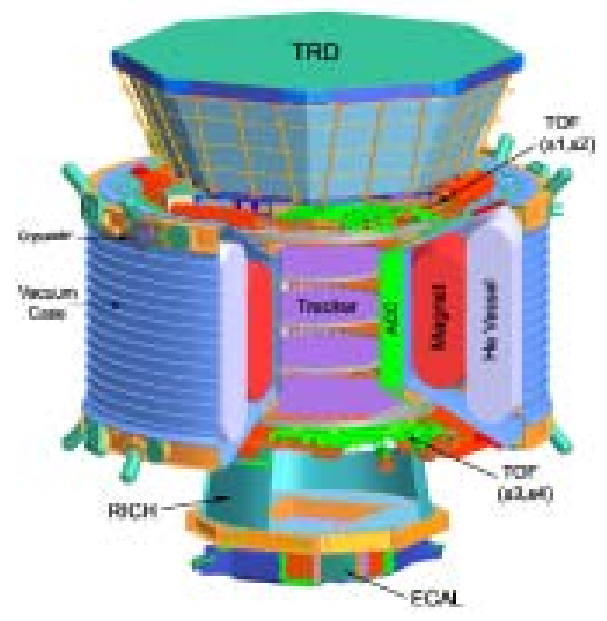

Fig. 1: The TRD in AMS-02

twenty $20 \mathrm{~mm}$ thick layers, with layers of $6 \mathrm{~mm}$ diameter straw tubes, filled with an 80\%:20\% $\mathrm{Xe}: \mathrm{CO}_{2}$ gas mixture, in between to detect the photons. In this way a rejection factor of $10^{3}-10^{2}$ for $\mathrm{p}^{+}$and $\mathrm{e}^{-}$can be achieved against $\mathrm{e}^{+}$and $\overline{\mathrm{p}}$ in the aforementioned momentum range. Combining the TRD rejection power with that of an electromagnetic calorimeter (ECAL in Fig. 1) located at the bottom of AMS-02 increases the $\mathrm{p}^{+}$rejection to the order of $10^{6}$ at $90 \% \mathrm{e}^{+}$efficiency, in this energy range.

\section{Mechanical Structure}

The TRD detector is supported by a conical octagon structure (width from $1.5 \mathrm{~m}$ at bottom to $2.2 \mathrm{~m}$ at top) built of a carbon fiber and aluminum honeycomb sandwich material, both for the sidewalls, with slots machined with a precision of $100 \mu \mathrm{m}$, and the top and bottom covers [2]-[5]. The dimensions are verified on a precision optical measuring machine. The octagon is supported from the magnet vacuum case and the Universal Support Structure (USS) which holds AMS-02 in the shuttle and on the ISS by an aluminun M-structure. Detailed finite element calculations have been performed to verify that the large TRD octagon structure 


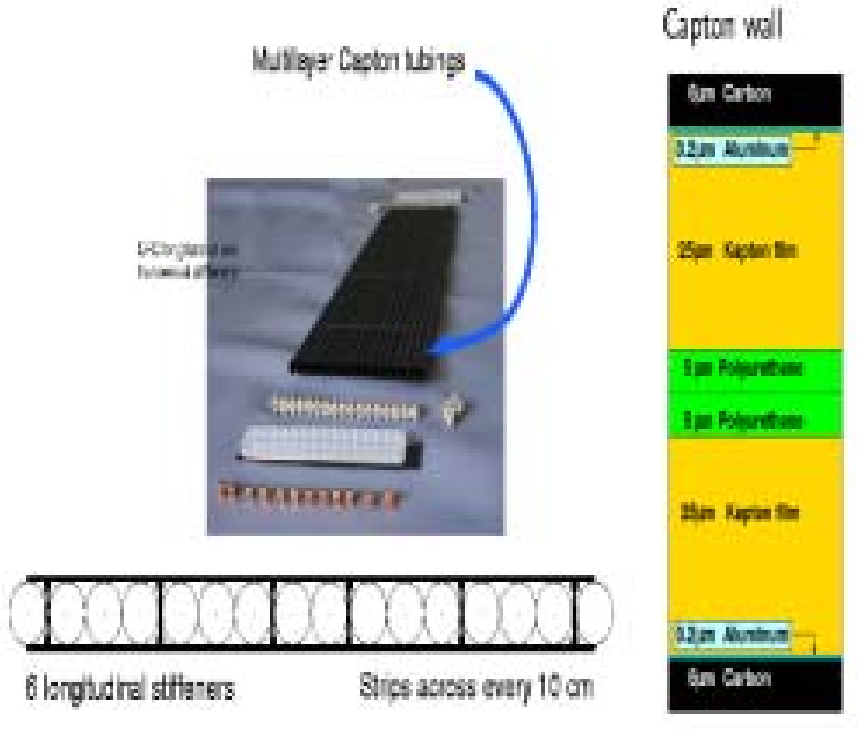

Fig. 2: Straw Tube Module: $166 \mathrm{~mm}$ tubes with $30 \mu \mathrm{m}$ W-Au wire

and support satisfies all dimensional and safety requirements. The straw tubes are built as modules of 16 tubes with $6 \mathrm{~mm}$ diameter. The 20 layers, each with $20 \mathrm{~mm}$ fleece, of modules are arranged in the conical octagon structure. The top and bottom 4 layers are oriented parallel to the AMS- $02 \times$ axis, which is the direction of the field in the magnet. The middle 12 layers are oriented along the perpendicular y direction. Thus the tubes provide tracking both in the bending and non-bending directions of the magnet as well as particle identification.

The length of the straw modules varies from $0.8 \mathrm{~m}$ to $2.0 \mathrm{~m}$. In all, there are 328 modules, for a total of 5248 straws. The wall material of the straws is a $72 \mu \mathrm{m}$ Kapton foil, whose structure is shown in Fig. 2. The sense wires are $30 \mu \mathrm{m}$ gold plated tungsten. The wires are held in the polycarbonate end pieces by crimping in $\mathrm{Cu}-\mathrm{Te}$ blocks. The radiator is a fleece of $10 \mu \mathrm{m}$ polyethylene/polypropylene fibers. The fiber sheets have a density of $0.06 \mathrm{~g} / \mathrm{cm}^{3}$ and are $5 \mathrm{~mm}$ thick. Before use, the material is cleaned with $\mathrm{CH}_{2} \mathrm{Cl}_{2}$. Measurements have shown that this is sufficient to attain the outgassing limit of $10^{-12} \mathrm{~g} / \mathrm{s} / \mathrm{cm}^{2}$ required by NASA.

\section{Electronics AND DAQ System}

The Data Acquisition (DAQ) system of the TRD is divided in two parts: The front end electronics, constituted of 5248 channels, which are mounted on the walls of the detector, and the first level of data acquisition which is hosted in two identical crates. The digitization of the signals from the straw tubes is done in the front end electronics. The crates hold the power supplies, the boards which collect and compress the data and the control of the whole TRD DAQ system.

\section{Module Production}

Each straw is pretested and accepted only with a He leakrate below $10^{-5} 1 \mathrm{mbar} / \mathrm{s} / \mathrm{m}$. Straw module production continues with gluing of 16 straws with their stiffeners. Then the endpieces are glued to the straws. After curing the glue, the wires are inserted, tensioned, and crimped into the CuTe blocks in a special machine. The wire tension is measured, then a preview test of the signal noise spectrum is made with $\mathrm{HV}$ and an $\mathrm{Ar} / \mathrm{CO}_{2}$ gas mixture. After these tests are passed, the final glue potting of the endpieces is done and the HV boards are mounted. This is followed by a serial test of gas tightness, dark current and corona, and the gas gain is measured as a function of high voltage with an $\mathrm{Fe}^{55}$ source and the $\mathrm{Ar} / \mathrm{CO}_{2}$ gas mixture. An X-ray measurement of the wire position is made on a subsample of the modules, as well as a long term test in vacuum of the gas gain.

\section{GAS SYSTEM}

The TRD contains $\mathrm{Xe} / \mathrm{CO}_{2}$ mixed 4:1 in volume. The gas has to be stored, mixed, and distributed through the TRD modules. The gas system to do this is divided into three parts. Box $\mathrm{S}$ stores the $\mathrm{Xe}$ and $\mathrm{CO}_{2}$ in separate vessels, which will contain $46 \mathrm{~kg}$ of $\mathrm{Xe}$ and $4 \mathrm{~kg}$ of $\mathrm{CO}_{2}, 50 \mathrm{~kg}$ in total. The gases are transferred in controlled amounts (by measuring partial pressures) to a mixing vessel, from which the mixture is released to box C. Box C contains redundant pumps to circulate gas through the TRD to ensure uniform gas properties. It also contains a $\mathrm{CO}_{2}$ analyzer and monitor tubes for measuring gas gain with an $\mathrm{Fe}^{55}$ source. Gas tightness is crucial for the operation of the TRD detector in space. The 160 flight modules produced so far all show a leak rate below $10^{-4} 1 \mathrm{mbar} / \mathrm{s} / \mathrm{m}$, which is sufficient for operating the TRD up to 10 years. Nonetheless, the volume of the TRD is divided into 41 separate gas circuits, each consisting of eight modules connected in series. Pressure sensors and valves located in the so-called manifolds can detect leaks and isolate a leaky segment.

\section{Monitoring AND CONTROL}

The monitoring and control crate for the gas system contains two redundant Universal Slow Control Modules (USCMs) which contain the monitor program which tests the status of the gas system against preset conditions and executes commands, which are stored in the form of decision tables. The USCMs are connected to the main Monitor and Control Computer of AMS via CAN-Bus, and to the gas system control electronics via a dedicated control bus. The gas control electronics consists of separate redundant Box S, Box C, and manifold control cards in the crate, and cards mounted on the manifolds for the pressure sensors. The cards monitor pressures, temperatures, gas composition and gain, and control the opening and closing of the valves for safety and gas mixing operations. 


\section{GAS GAIN CONTROL}

To achieve the requested performance the gas gain, $\mathrm{G}$, has to be controlled carefully. This has dictated severe constrains to our detector. The effect of mechanical wire displacement is for example extremely important. A $100 \mu \mathrm{m}$ wire displacement off the center causes a change in the gas amplification by $1.5 \%$.

The percentage of the two gas $\mathrm{Xe} / \mathrm{CO}_{2}$ is higly determining the amplification

$$
\frac{d G}{G d f}=\frac{8 \%}{1 \% \mathrm{CO}_{2}}
$$

where $\mathrm{f}$ is the $\mathrm{CO}_{2}$ percentage in the gas mixture. A variation of ony $1 \%$ in $\mathrm{CO}_{2}$ content implies a $8 \%$ variation on gas gain

The gas density, $\rho$, also, has to be carefully controlled:

$$
\frac{d G}{G d \rho}=\frac{5.5 \%}{1 \% \rho}
$$

a variation of $1 \%$ in the density implies a $5 \%$ variation in gas gain. The same variation is caused by a temperature gradient of $2.7 \mathrm{~K}$ assuming a constant gas volume.

Another important element is the control of applied voltage U.

$$
\frac{d G}{G d U}=\frac{1 \%}{1 V}
$$

a variation of $1 \mathrm{~V}$ in the applied voltage $\mathrm{U}$ implies a $2 \%$ variation in gas gain. In the design and realization of this dector these effects have been taken in account.

\section{VERIFICATION OF TRD PERFORMANCE}

The TRD is described in [2]-[5]. To verify the performance, a 20 layer prototype was built with two 16 tube modules staggered side by side in each layer. This was used for a beam test at CERN. In 16 layers the tubes ran horizontally, and in 4 layers the tubes ran vertically. Aside from length, the tubes and radiator were identical to those of the flight TRD. They were filled with an $80 \%: 20 \% \mathrm{Xe}: \mathrm{CO}_{2}$ gas mixture at 1 bar absolute pressure. Runs were taken at 15 different beam energies at test beams at CERN. Over 3 million events were recorded: $\mathrm{p}^{+}$at $15-250 \mathrm{GeV}, \mathrm{e}^{-}, \mu^{-}$and $\pi^{-}$at $20-100$ $\mathrm{GeV}$. Particles were identified by Cerenkov counters and by penetration of an iron beam dump at the end of the beam line. A glance at energy spectra of protons, pions and electrons, as in Fig.3, clearly shows the effect of transition radiation and the dependence of TR on the Lorentz factor $\gamma$.

\section{CAlibration}

A tube by tube intercalibration with protons and muons was done to equalize the signals from each tube to a standard value. This was done to correct for differences in the electronics and mechanical construction of the different tubes. For each run, a Landau fit was done to each tube's energy deposit spectrum to determine its most probable value, and these were used to prepare intercalibration tables of the tubes, run by run, and then summed for all runs, using overlapping tubes. The intercalibrations are accurate to the $1 \%$ level. Pressure and

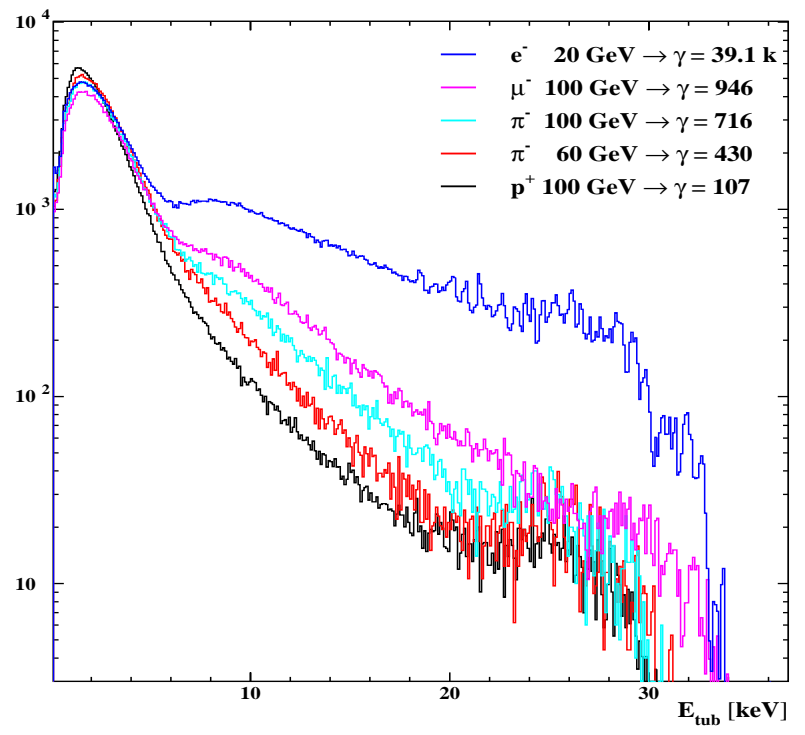

Fig. 3: Energy spectra recorded with particle at varoius Lorentz factors [4]
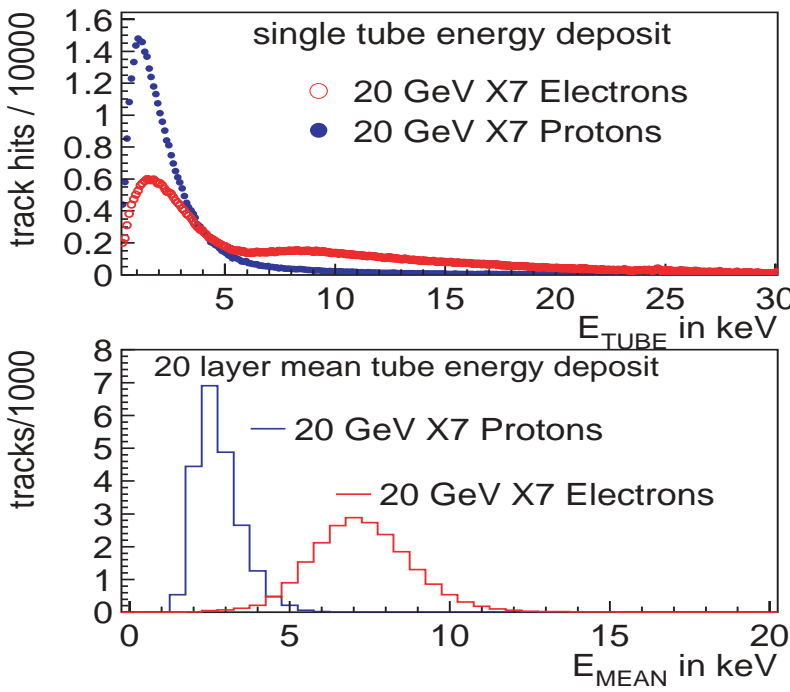

Fig. 4: Energy deposition of electrons and protons in a single tube (top) and in 20 layers (bottom)

temperature were monitored for gas density corrections between runs. At the standard density of $4.46 \times 10^{-3} \mathrm{~g} / \mathrm{cm}^{3}$ with $\mathrm{HV}$ at $1470 \mathrm{~V}$ for a gas gain of 5000 an increase of density of $1 \%$ leads to a decrease of gas amplification of $5.5 \%$. The correction is accurate to $1.5 \%$. $\mathrm{Fe}^{55}$ spectra were taken between runs on the first and last layers to calibrate the energy deposition by photons to the ADC scale. $9.09 \pm 0.05 \mathrm{eV}$ of photon energy corresponded to one 12 bit ADC bin.

The calibrations were done both for protons and for muons, with good agreement. 
A TRD protype, built with 20 layers of straw tubes, has been calibrated and its performance measured in test beams with $\mathrm{p}^{+}, \mathrm{e}^{-}, \mu^{-} \pi^{-}$in the energy range from 3 to $250 \mathrm{GeV} / \mathrm{c}$ and compared with Monte-Carlo predictions. The rejection factors achieved is in the range of 2000-140 for protons in an energy range of $15-250 \mathrm{GeV} / \mathrm{c}$. This requirement is fundamental to reach the precision in positron spectroscopy over the dominating proton background to observe the positrons originated from neutralino annihilation.

\section{ACKNOWLEDGMENT}

I would like to thank all my colleagues and technical staff in the collaboration for all their efforts, in particulary J.Burger (MIT) and Th. Siedenburg (Aachen).

\section{REFERENCES}

[1] U.Becker,these proceedings. S.Gentile,"The Alpha Magnetic Spectrometer on the International Space Station", Proceedings of 28th International Cosmic Ray Conference, (Tsukuba),2003.

[2] F.Hauler, these proceedings. J.Burger and S.Gentile," The AMS-02 TRD for the International Space Station", Proceedings of 28th International Cosmic Ray Conference, (Tsukuba),2003.

[3] J.Burger and S.Gentile," The Performance of the AMS-02 TRD", Proceedings of 28th International Cosmic Ray Conference, (Tsukuba),2003.

[4] Joerg Orboeck, "The final 20-Layer-Prototype for the AMS Transition Radiation Detector: Beamtests, Data-analyis,Mc-Studies", PhD. Thesis. I Physikalisches Institut of RWTH-Aachen, May 2003

[5] T. Siedenburg, "A Transition Radiation Detector for AMS", Nucl.Phys. B (Proc.Suppl.), vol. 113 (2002), 154-158. 